\title{
EfeCto del Sitio de PROCEDENCIA SOBRe LOS ATRIBUtos DE LAS SEMILLAS DE CAKILE EDENTULA (BRASSICACEAE), ESPECIE ESTABILIZADORA DE DUNA COSTERA
}

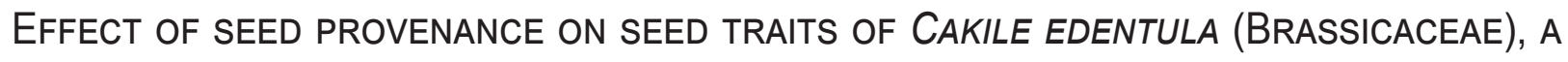 COASTAL DUNE STABILIZING SPECIES}

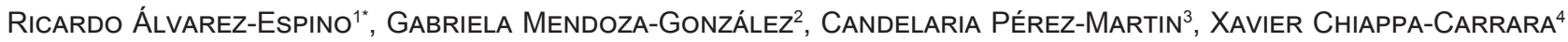 \\ ${ }^{1}$ Facultad de Ciencias, UMDI Sisal, Universidad Nacional Autónoma de México, Mérida, Yucatán, México. \\ ${ }^{2}$ CONACYT-Facultad de Ciencias, UMDI Sisal, Universidad Nacional Autónoma de México, Mérida, Yucatán, México. \\ ${ }^{3}$ Banco de Germoplasma, Centro de Investigación Científica de Yucatán, A. C., Yucatán, México. \\ ${ }^{4}$ Escuela Nacional de Estudios Superiores Mérida, Universidad Nacional Autónoma de México, Mérida, Yucatán, México.
}

*Autor de correspondencia: r.alvarez.espino@gmail.com

\begin{abstract}
Resumen
Antecedentes: Los rasgos morfológicos y el comportamiento germinativo de las semillas varían dentro de una especie en respuesta a múltiples factores. El conocimiento de esta variabilidad es importante para entender la adaptación de las especies a las condiciones ambientales. Preguntas: ¿La procedencia de las semillas de Cakile edentula afecta los atributos de las semillas? ¿El clima de los sitios de procedencia influye en su variabilidad? ¿La presencia de luz afecta la germinación de las semillas?

Especie de estudio: Cakile edentula (Bigelow) Hook.

Sitio de estudio: Vegetación de duna costera en la costa norte de la Península de Yucatán, en agosto y octubre de 2017.

Métodos: Se colectaron semillas de Cakile edentula en distintas localidades de la costa norte de la Península de Yucatán. En laboratorio se evaluó si la procedencia de las semillas influyó en algunos atributos como peso, contenido de humedad, viabilidad y comportamiento germinativo.

Resultados: El sitio de procedencia afectó significativamente el peso y el contenido de humedad de las semillas de C. edentula. Además, las semillas de sitios cálidos y húmedos germinan rápidamente y en mayor proporción que las semillas provenientes de sitios cálidos y secos de la Península de Yucatán. Las semillas de C. edentula germinan preferentemente en oscuridad, independientemente del sitio de procedencia. Conclusiones: Algunos atributos de las semillas de Cakile edentula son afectados por el sitio de procedencia. Las evidencias sugieren que probablemente la variación climática influye en las características iniciales de la historia de vida de esta especie de duna costera.
\end{abstract}

Palabras clave: Cakile edentula, duna costera, fotoblastismo negativo, germinación.

\begin{abstract}
Background: The morphological traits and germinative behavior of seeds vary within a species in response to multiple factors. Knowledge of this variability is important in understanding adaptation of species to environmental conditions.

Question: Does the provenance of seeds of Cakile edentula affect seed traits? Does the climate of the sites of origin of the seeds influence their variability? Does the presence of light affect seed germination?

Study species: Cakile edentula (Bigelow) Hook.

Study site: Coastal dune vegetation on the northern coast of the Yucatan Peninsula in August and October 2017.

Methods: Seeds of Cakile edentula were collected in different locations on the northern coast of the Yucatan Peninsula. Under laboratory conditions it was assessed whether the origin of the seeds influenced some traits such as seed mass, moisture content, viability and germinative behavior.

Results: Seed provenance significantly affected seed mass and moisture content in C. edentula. Moreover, the seeds of $C$. edentula collected from hot and humid sites germinate quickly and in greater proportion than the seeds from warm and dry sites of the Yucatan Peninsula. The seeds of $C$. edentula germinate preferably in darkness, regardless site of provenance.

Conclusions: Some seed traits of C. edentula are affected by the site of provenance. Evidence suggests that climatic variation is likely to influence the initial characteristics of the life history in this coastal dune species.
\end{abstract}

Keywords: Cakile edentula, coastal dune, germination, negative photoblastism.

This is an open access article distributed under the terms of the Creative Commons Attribution License CCBY-NC (4.0) international. cC) (7) \&
https://creativecommons.org/licences/by-nc/4.0/ 
El nicho germinativo es determinante para la colonización y el mantenimiento de las poblaciones (Cochrane et al. 2011). La amplitud del nicho de germinación puede influenciar el intervalo geográfico de la distribución de las especies. De esta manera, especies con un amplio nicho de germinación tendrían un amplio intervalo de distribución geográfica; mientras que especies con nichos de germinación estrechos tendrían un intervalo geográfico menor. Aunque poca evidencia empírica respalda esta idea (Thompson \& Ceriani 2003, Cochrane et al. 2011), se ha encontrado que, para muchas especies, los rasgos ecológicos y fisiológicos, así como las señales de germinación controladas por la temperatura son cruciales en determinar los mecanismos de latencia, germinación y establecimiento de las plántulas (Cochrane et al. 2014, Carta et al. 2016). En este sentido, se ha encontrado que el ambiente de maduración de las semillas (i.e., el clima local) se correlaciona con el tipo y grado de latencia en Hypericum elodes (Hypericaceae; Carta et al. 2016) así como en cinco especies de Cyperaceae (Fernández-Pascual et al. 2015), y también es el principal predictor del porcentaje de germinación en 11 especies de Caryophyllaceae (Zani \& Müller 2017).

Los distintos atributos de las semillas han sido funcionalmente relacionados con los procesos de dispersión, pero también revelan propiedades de su persistencia en el suelo (Fenner \& Thompson 2005). Se han establecido correlaciones entre el peso, el tipo de dispersión, la producción de semillas, la formación de bancos de semillas, la germinación y el tamaño inicial de las plántulas (Westoby et al. 2002, Jiménez-Alfaro et al. 2016). Mientras que atributos como la tolerancia a la desecación, el contenido de agua y aceites han sido relacionadas entre sí, también se han correlacionado con gradientes latitudinales (Jiménez-Alfaro et al. 2016). Adicionalmente, el tipo de latencia y la conducta germinativa se han vinculado principalmente con adaptaciones genéticas que pueden ser afectadas por las condiciones climáticas a través de efectos maternos (Rosbakh \& Poschlod 2015, Jiménez-Alfaro et al. 2016).

La variación existente en los atributos de las semillas entre poblaciones, respalda la capacidad de las plántulas para emerger, crecer y establecerse (Cochrane et al. 2015) y podrían aminorar los efectos del clima sobre la adecuación y persistencia de las plantas. Esta variación en los atributos tanto dentro como entre especies está fuertemente influenciada por el origen geográfico de las variables ambientales de los sitios de procedencia (Guo et al. 2010). Consecuentemente, se podría predecir el riesgo en el mantenimiento de las poblaciones ante disturbios y cambios ambientales mediante el análisis de la variación de los atributos de las semillas, a través del intervalo de distribución de las especies (Walck et al. 2011).

La región donde se desarrolló el presente estudio ocupa el segundo lugar con mayor número de especies endémicas de la flora costera de México, donde las comunidades vegetales aportan importantes servicios ecosistémicos (Torres et al. 2010). Funcionan como barreras de protección a la acción de vientos y mareas, contribuyen a la formación de suelo y brindan sitios de refugio, alimento y protección de numerosas especies de insectos, reptiles, aves y mamíferos (Torres et al. 2010, Jiménez-Orocio et al. 2015). Las comunidades vegetales dentro de la primera línea de costa son altamente vulnerables a los efectos del clima debido a que se encuentran en una región con baja topografía lo cual las enfrenta a escenarios de cambio climático y de incremento de nivel del mar.

Nuestro objetivo principal fue evaluar el efecto del sitio de procedencia de las semillas de Cakile edentula (Bigelow) Hook. (Brassicaceae) sobre los principales atributos ecológicos y físiológicos de las semillas, como son el tamaño, el contenido de humedad a la dispersión (y tolerancia a la desecación), la viabilidad y la capacidad germinativa de las semillas. Se explora si las condiciones climáticas de los distintos sitios de procedencia de $C$. edentula en la Península de Yucatán podrían influir en la variación de los atributos de las semillas.

\section{Materiales y métodos}

Sitio de estudio. El estudio se realizó en la costa norte de la Península de Yucatán, incluyendo a los estados de Quintana Roo y Yucatán, así como diferentes tipos de clima, desde los áridos y semiáridos al noroeste de la Península hasta los cálidos subhúmedos al noreste (según la clasificación de Köppen modificada por García 2004). Los sitios de colecta de las semillas de C. edentula fueron: 1) Celestún, 2) Palmar, 3) Chuburná, 4) Sisal, 5) El Cuyo y 6) Holbox. Estos sitios representan un gradiente longitudinal a lo largo de la distribución de $C$. edentula en la Península de Yucatán, en donde el clima es variable. Se muestran detalles de ubicación y clima de los sitios de estudio en la Tabla 3.

Especie estudiada. Cakile edentula (Brassicaceae) es una planta pionera que crece en los primeros 5-10 $\mathrm{m}$ de la primera línea de costa en vegetación de matorral y duna costera. Se distribuye principalmente en las costas de Norteamérica. Tiene hojas y tallos suculentos, aunque se tornan leñosos con el tiempo. Las ramas crecen cerca de la base hacia afuera $\mathrm{y}$ hacia arriba, las hojas maduras regularmente son gruesas, carnosas, crenadas y ligeramente dentadas (Maun et al. 1990). Sus flores son blancas de $10-20 \mathrm{~mm}$. La infrutescencia es en racimo, los frutos son indehiscentes y tienen dos segmentos de diferente morfología. Un segmento distal elipsoide que es deciduo y un segmento proximal piramidal que se queda anclado al racimo. Regularmente tienen una semilla por segmento, oval o elipsoide de ca. $5 \mathrm{~mm}$ de longitud, con una cubierta lisa de color bronce. La variación que presenta $C$. edentula en la arquitectura del fruto influye en mayor medida en la distancia a la que se dispersan las semillas más que en otros atributos como el peso y la germinación (Donohue 1988). Por esta razón, en el presente estudio solamente se utilizaron semillas de la parte distal de los frutos.

Colecta de semillas. En cada sitio se colectaron alrededor de 500 frutos maduros de al menos 20 individuos saludables de C. edentula entre agosto y octubre de 2017. Los frutos fueron 
seleccionados aleatoriamente dentro de las inflorescencias para disminuir el efecto de la posición sobre los atributos de las semillas. En cada sitio de colecta se registraron las coordenadas geográficas con GPS (Garmin MAP 78). Las semillas fueron separadas de la parte distal de los frutos y almacenadas en bolsas de papel y en oscuridad hasta que fueron procesadas. 300 semillas por sitio de procedencia fueron aleatoriamente seleccionadas y utilizadas en los ensayos.

Atributos de las semillas. Se estimó el peso promedio (mg) de las semillas $(\mathrm{n}=30)$ de cada sitio de colecta utilizando una balanza analítica (Ohaus Adventurer, precisión 0.0001 g). Se determinó el contenido de humedad inicial en semillas recientemente colectadas $(\mathrm{n}=20)$ mediante la diferencia del peso fresco y seco de las semillas. Se utilizó un horno a 103 ${ }^{\circ} \mathrm{C}$ por $18 \mathrm{~h}$ para el secado de las semillas (ISTA 2015). Para determinar la tolerancia a la desecación de las semillas y su comportamiento en condiciones de almacén se utilizaron los métodos descritos por Hong \& Ellis (1996). En una muestra de semillas de cada población o sitio de procedencia $(n=80)$ se disminuyó el contenido de humedad $(\mathrm{CH})$ de las semillas con gel de sílice, partiendo del contenido de humedad inicial. Después de que las semillas alcanzaron un porcentaje de $\mathrm{CH}$ de $5-6 \%$, fueron almacenadas a $-20{ }^{\circ} \mathrm{C}$ por 3 meses. Se estimó la viabilidad de las semillas tanto antes como después de la prueba de tolerancia a la desecación con pruebas de tetrazolio, de acuerdo con los métodos de la ISTA (2015). Se utilizaron dos concentraciones de 0.1 y $1 \%$ de Cloruro de 2,3,5 trifeniltetrazolio, sin embargo, después de múltiples ensayos se determinó que la concentración de $1 \%$ fue más exitosa en teñir las semillas viables de $C$. edentula. Las semillas fueron embebidas antes de la prueba con agua destilada durante $16 \mathrm{~h}$, después fueron colocadas en la solución de cloruro de tetrazolio por $16 \mathrm{~h}$ en oscuridad. Se evaluó el porcentaje de semillas viables de cada muestra $(n=20)$ con el apoyo de un microscopio estereoscópico (Leica EZ4 HD). Se consideró una semilla viable al observar una tonalidad roja en el embrión.

Germinación. Para las pruebas de germinación se utilizaron cajas de Petri de $50 \mathrm{~mm}$, con un sustrato de vermiculita saturada con agua destilada. Cada caja de Petri contenía 10 semillas y se utilizaron 5 repeticiones para cada sitio de procedencia de las semillas. Las cajas se mantuvieron en una cámara de crecimiento Binder $\mathrm{Gmbh}^{\circledR}$ modelo KFB720 a temperatura constante de $30{ }^{\circ} \mathrm{C}$ y humedad relativa de $60 \%$. Se utilizaron dos tratamientos: luz y oscuridad (5 repeticiones por tratamiento). En el tratamiento de presencia de luz, se utilizó un fotoperiodo de $12 \mathrm{~h}$ luz/ $12 \mathrm{~h}$ oscuridad mientras que, en el tratamiento de oscuridad, las cajas de Petri fueron cubiertas por dos capas de papel de aluminio. Las cajas de Petri se revisaron cada 3 días para contar el número de semillas germinadas, considerando una semilla germinada al observar la emergencia de la radícula.

Variables climáticas. Se obtuvieron 19 variables bioclimáticas de los sitios de procedencia de las semillas a través de la base de datos de WorldClim (www.worldclim.com). Estas variables contienen información global en formato raster con una resolución espacial de $0.0083^{\circ}\left(\sim 1 \mathrm{~km}^{2}\right)$ y representan condiciones bioclimáticas relacionadas con precipitación y temperatura (Tabla 2). Para analizar la variación climática entre los sitios de procedencia de las semillas de C. edentula, se realizaron análisis de componentes principales (PCA, por sus siglas en inglés). Inicialmente, se disminuyó el número de variables mediante la prueba de Pearson $(P<0.05)$, eliminando aquellas altamente correlacionadas $\left(r^{2}>0.8\right)$ para disminuir la colinealidad en los datos. Posteriormente, se realizó un PCA con 7 variables bioclimáticas (Tabla 3) y se examinó la magnitud y dirección de los coeficientes de las variables originales. A mayor valor absoluto del coeficiente, más importante fue la variable bioclimática correspondiente en la ordenación de los sitios de procedencia.

Adicionalmente, para conocer la asociación de los atributos de las semillas con las variables bioclimáticas de los sitios de procedencia, se utilizaron correlaciones bivariadas de Pearson.

Análisis de datos. Se comparó el peso de las semillas entre los sitios de procedencia de C. edentula con análisis de varianza (ANOVA) de una vía y pruebas de comparaciones múltiples de Tukey cuando existieron diferencias significativas. El CH se comparó entre sitios de procedencia con Modelos Lineales Generalizados (GLM, por sus siglas en inglés) previa transformación arcoseno del porcentaje de humedad de las semillas. La viabilidad de las semillas se comparó entre sitios de procedencia con GLM, utilizando una distribución binomial y una función de enlace logit. Por otro lado, se analizó el efecto de la procedencia de las semillas, de la presencia de luz y de la interacción de ambos factores sobre el porcentaje final de germinación, mediante GLM, considerando una distribución binomial (semilla germinada/no germinada) y una función de enlace logit. Se realizaron pruebas de comparaciones múltiples de Tukey cuando existió un efecto significativo en la procedencia de las semillas. Todos los análisis estadísticos fueron realizados con el programa SPSS (IBM versión 21.0).

\section{Resultados}

Atributos de las semillas. El peso de las semillas de C. edentula presentó diferencias significativas en semillas de distinta procedencia $(F=7.39, P<0.001$; Tabla 1$)$. En general, se observó alta variabilidad en los valores obtenidos para este atributo (Tabla 1). Las semillas con mayor peso fueron las que provenían de la localidad de Palmar $(7.18 \pm 0.27 \mathrm{mg})$, mientras que las semillas de menor peso fueron las procedentes de la localidad de El Cuyo $(4.57 \pm 0.22 \mathrm{mg})$. El peso promedio de las semillas entre ambos sitios fue significativamente diferente, al igual que con el resto de los sitios de colecta $(P<0.05$, Tabla 1). Las semillas de Celestún, Sisal, Chuburná y Holbox tuvieron pesos similares (5.5-6.11 mg), y no se observaron diferencias significativas entre estos sitios de procedencia $(P>0.05$, Tabla 1$)$. De la misma manera, el sitio de precedencia de las semillas tuvo un efecto significativo en el contenido de humedad $(\mathrm{CH})$ de las semillas 
Tabla 1. Atributos de semillas de Cakile edentula de distinta procedencia en la Península de Yucatán, México. Peso y contenido de humedad inicial (promedio \pm error estándar) y viabilidad inicial de semillas. Letras diferentes indican diferencias significativas entre sitios de procedencia (comparaciones múltiples de Tukey, $P<0.05$ ).

\begin{tabular}{lccc}
\hline Sitio de procedencia & Peso de la semilla (mg) & Contenido de humedad (\%) & Viabilidad (\%) \\
\hline Celestún & $5.56 \pm 0.29 \mathrm{ab}$ & $11.32 \pm 0.15 \mathrm{a}$ & $30 \mathrm{a}$ \\
Palmar & $7.18 \pm 0.27 \mathrm{c}$ & $9.61 \pm 0.58 \mathrm{a}$ & $30 \mathrm{a}$ \\
Sisal & $6.03 \pm 0.51 \mathrm{bc}$ & $30.64 \pm 2.63 \mathrm{~b}$ & $40 \mathrm{a}$ \\
Chuburná & $5.55 \pm 0.21 \mathrm{ab}$ & $11.41 \pm 0.12 \mathrm{a}$ & $55 \mathrm{a}$ \\
El Cuyo & $4.57 \pm 0.22 \mathrm{a}$ & $33.95 \pm 1.45 \mathrm{~b}$ & $60 \mathrm{a}$ \\
Holbox & $6.11+-0.30 \mathrm{bc}$ & $34.00 \pm 1.72 \mathrm{~b}$ & $50 \mathrm{a}$ \\
Probabilidad & $P<0.001$ & $P<0.001$ & $P=0.964$ \\
\hline
\end{tabular}

$(F=99.57, P<0.001$; Tabla 1$)$. Las semillas colectadas en El Cuyo, Holbox y Sisal formaron un grupo, y tuvieron valores significativamente más altos $(\mathrm{CH}=30-34 \%$; Tabla 1$)$ que las semillas de Palmar, Celestún y Chuburná, que formaron otro grupo $(\mathrm{CH}=9-11 \%$; Tabla 1$)$. Por otro lado, la viabilidad de las semillas recientemente colectadas no presentó diferencias significativas $\left(\chi^{2}=0.982, P=0.964\right)$ entre distintos sitios de procedencia (Tabla 1) y se observaron valores de 30-60\% de semillas viables en las pruebas de tetrazolio. Se realizaron pruebas de tolerancia a la desecación con las semillas procedentes de Celestún, Chuburná y Palmar y se encontró que $30-45 \%$ de las semillas mantienen la viabilidad con valores de $\mathrm{CH}=5 \%$ después de 3 meses de almacenamiento a temperaturas constantes de $-20{ }^{\circ} \mathrm{C}$.

Germinación. Las semillas de C. edentula inician la germinación después de $24 \mathrm{~h}$ de la siembra y después de 5-7 días la germinación se detiene (Figura 1). Se encontró que

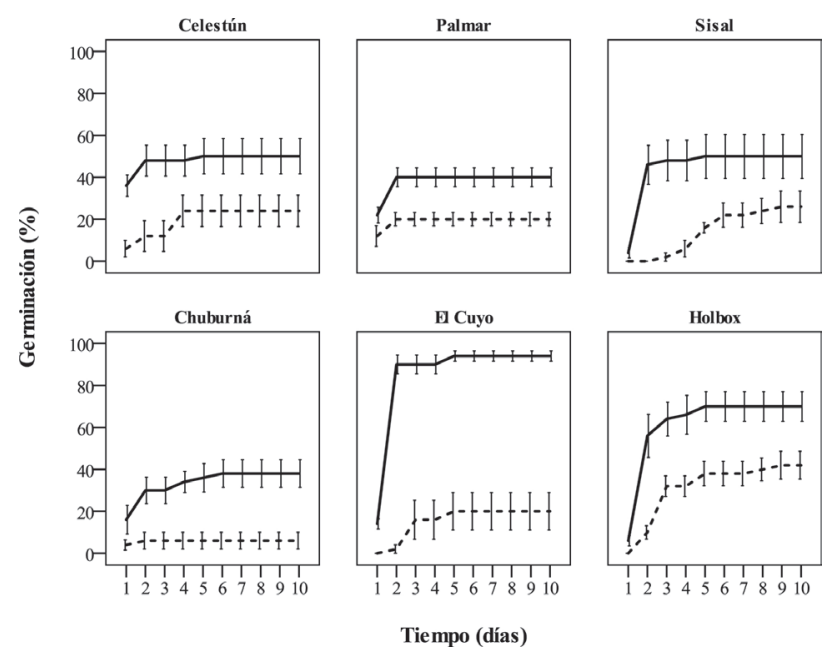

Figura 1. Germinación acumulativa (promedio \pm error estándar) de las semillas de Cakile edentula de distintos sitios de procedencia en la Península de Yucatán, México. Tratamiento de luz (línea discontinua) y oscuridad (línea continua). la luz inhibe la germinación de las semillas de $C$. edentula (Figuras 1,2). Estos patrones fueron similares en los distintos sitios de procedencia, sin embargo, la proporción de semillas germinadas si presentó variaciones. En el experimento de germinación bajo condiciones controladas, se encontró que el sitio de procedencia de las semillas $\left(\chi^{2}=36.74, P<0.001\right)$, la presencia de luz $\left(\chi^{2}=73.21, P<0.001\right)$ y la interacción de ambos factores $\left(\chi^{2}=18.16, P<0.01\right)$ afectaron de manera significativa el porcentaje final de germinación en esta especie (Figura 2). Los porcentajes finales de germinación (Figuras 1,2) en condiciones de oscuridad de las semillas procedentes de El Cuyo (90-100 \%) y Holbox (50-90\%) fueron los más altos del estudio. La germinación entre estos dos sitios fue significativamente diferente, así como con el resto de los sitios de procedencia $(P<0.05$; Figura 2$)$. En las mismas condiciones de oscuridad, las semillas de Celestún, Palmar, Sisal y Chuburná germinaron en menor proporción (20-70\%), y no fueron significativamente diferentes $(P>$

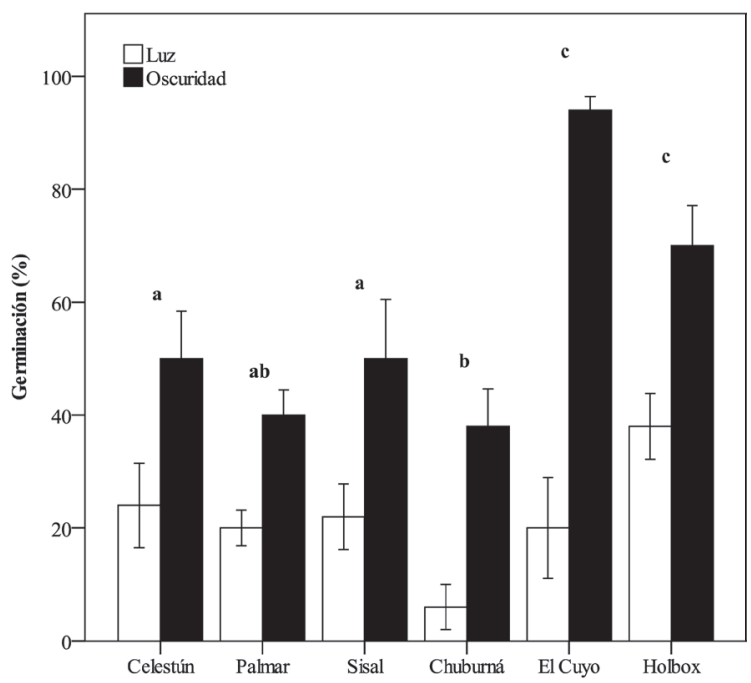

Figura 2. Porcentaje final de germinación (promedio \pm error estándar) de semillas de $C$. edentula de diferentes procedencias en condiciones de luz y oscuridad. Letras diferentes indican diferencias significativas (comparaciones múltiples de Tukey, $P<0.05$ ). 
Tabla 2. Variables bioclimáticas utilizadas para la caracterización ambiental de los sitios de estudio

\begin{tabular}{|c|c|}
\hline Código & Variables Bioclimáticas \\
\hline Biol & Temperatura media anual \\
\hline Bio2 & $\begin{array}{l}\text { Rango de temperatura diurno medio } \\
\text { (Temp. Máxima - Temp. Mínima) }\end{array}$ \\
\hline Bio3 & Isotermalidad $(\mathrm{Bio} 2 / \mathrm{Bio} 7)(\times$ 100) \\
\hline Bio4 & $\begin{array}{l}\text { Estacionalidad de temperatura } \\
(\text { desviación estándar } \times 100)\end{array}$ \\
\hline Bio5 & Temperatura máxima del mes más cálido \\
\hline Bio6 & Temperatura mínima del mes más frío \\
\hline Bio7 & Rango de temperatura anual (Bio5 - Bio6) \\
\hline Bio8 & Temperatura media del trimestre más lluvioso \\
\hline Bio9 & Temperatura media del trimestre más seco \\
\hline Bio10 & Temperatura media del trimestre más cálido \\
\hline Bio11 & Temperatura media del trimestre más frío \\
\hline Bio12 & Precipitación total anual \\
\hline Bio13 & Precipitación del mes más lluvioso \\
\hline Bio14 & Precipitación del mes más seco \\
\hline Bio 15 & $\begin{array}{l}\text { Estacionalidad de la precipitación } \\
\text { (coeficiente de variación) }\end{array}$ \\
\hline Bio16 & Precipitación del trimestre más lluvioso \\
\hline Bio17 & Precipitación del trimestre más seco \\
\hline Bio18 & Precipitación del trimestre más cálido \\
\hline Bio19 & Precipitación del trimestre más frío \\
\hline
\end{tabular}

0.05 , Figura 2). De manera generalizada, la presencia de luz inhibe la germinación de las semillas de $C$. edentula, aunque las semillas más afectadas por esta condición fueron las procedentes de Chuburná, que presentaron los valores significativamente más bajos $(0-20 \%, P<0.05$, Figuras 1,2$)$. El porcentaje de germinación final en presencia de luz no fue significativamente diferente en semillas de Celestún, Palmar, Sisal, El Cuyo y Holbox, que mostraron valores intermedios $(0-50 \%, P>0.05$, Figura 2$)$.

Variables climáticas. Se utilizaron inicialmente 19 variables bioclimáticas de los sitios de colecta de semillas de Cakile edentula obtenidas de WorldClim (Tabla 2) y se eliminaron aquellas altamente correlacionadas. El Análisis de Componentes Principales (PCA) realizado con 7 variables bioclimáticas (Tabla 3 y Figura 3) explicó $97.9 \%$ de la variación en los sitios de procedencia dentro los dos primeros ejes principales (Figura 3). Las variables de mayor importancia en el eje principal 1 fueron la precipitación del trimestre más seco y la precipitación del trimestre más frío (con cargas positivas). Se observaron valores relativamente más altos de estas variables en los sitios de colecta de El Cuyo y Holbox (Tabla 3). Ambos sitios formaron un grupo separado del resto de los sitios de colecta sobre el eje 1 del PCA (Figura 3). Las variables temperatura media del trimestre más frio y temperatura media del trimestre más seco también fueron importantes (con cargas negativas) en la ordenación de los sitios de Celestún, Palmar, Sisal y Chuburná hacia el lado izquierdo de los cuadrantes del PCA (Figura 3). Los valores relativamente más altos en las variables antes mencionadas y particularmente los valores más altos en la precipitación del trimestre más lluvioso (Tabla 3) separaron ligeramente a Celestún de los sitios del Palmar, Sisal y Chuburná sobre el eje 2 del PCA (Figura 3). De manera general, la variabilidad climática observada en la ordenación de las variables bioclimáticas representa el gradiente longitudinal de los sitios de colecta de $C$. edentula que va del extremo noroeste (Celestún) al noreste (Holbox) de la Península de Yucatán. Por otro lado, no se encontró una asociación significativa entre el peso, contenido de humedad o la viabilidad de las semillas y las variables bioclimáticas de los sitios de procedencia $(P>$ 0.05 , Tabla 4). Por el contrario, se encontró una asociación positiva y significativa entre el porcentaje de germinación de las semillas y la temperatura máxima del mes más cálido (Bio5) $(r=0.82, P=0.43$; Tabla 4$)$ y con la precipitación del trimestre más frío (Bio 19) $(r=0.80, P=0.47$; Tabla 4). Es decir, en los sitios en donde la temperatura de los meses cálidos fue mayor, pero que en los meses fríos hubo una mayor precipitación, se observó una germinación más alta. En este estudio, representados por los sitios de colecta de El Cuyo y Holbox. Estos resultados fueron consistentes en los análisis multivariados y univariados.

\section{Discusión}

La emergencia de plántulas está frecuentemente sincronizada con los cambios estacionales en el ambiente (Baskin \& Baskin 1998, Fenner \& Thompson 2005). La germinación de muchas especies ocurre poco después de la dispersión, mien-

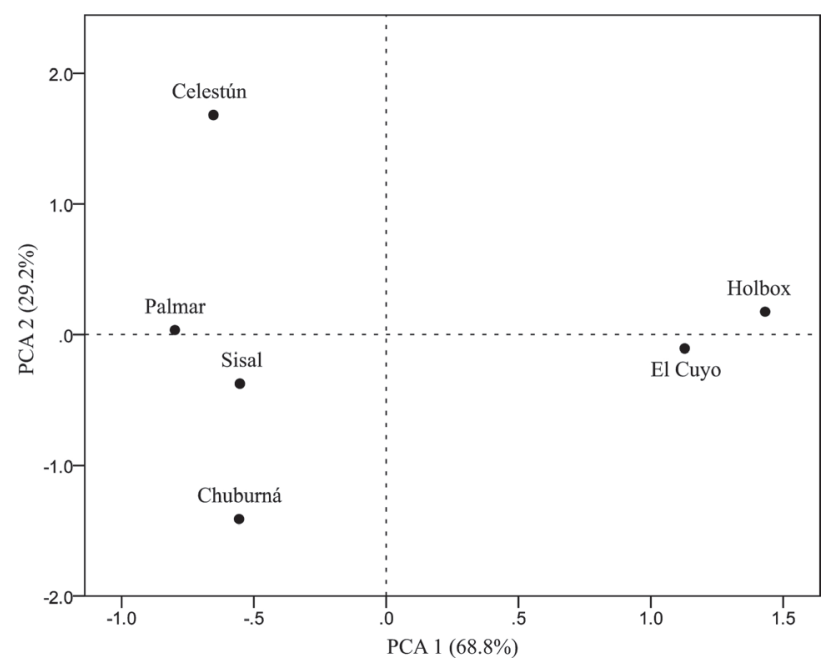

Figura 3. Análisis de Componentes Principales de los sitios de procedencia de las semillas de C. edentula en la Península de Yucatán, México, basado en variables bioclimáticas de temperatura y precipitación. 


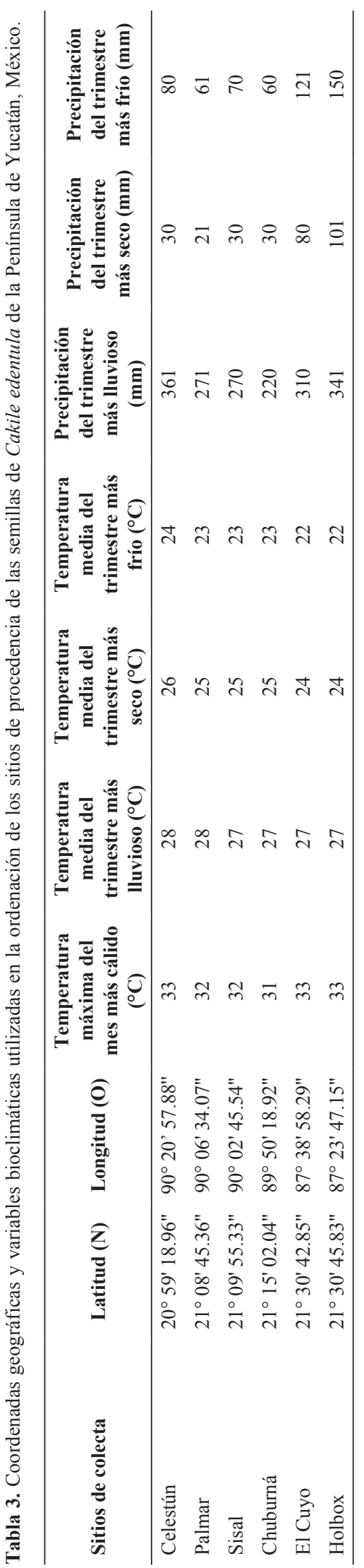

tras que algunas otras la retrasan a través de la latencia hasta que se alcancen las condiciones favorables para que las plántulas incrementen sus posibilidades de crecer y establecerse (Venable \& Brown 1988). En ambientes con condiciones variables y bajo un rápido cambio climático, el amortiguamiento sería influenciado por los atributos iniciales de la historia de vida, así como por la variación entre poblaciones (Cochrane et al. 2015, Jiménez-Alfaro et al. 2016). Nuestros resultados apoyan lo encontrado por otros investigadores que reportan diferencias a nivel poblacional en los atributos de las semillas (Fernández-Pascual et al. 2015, Torres-Martínez et al. 2017, Viveros-Viveros et al. 2017, Wu et al. 2018). El sitio de procedencia afectó el peso, contenido de humedad y germinación de las semillas de Cakile edentula. Asimismo, la presencia de luz inhibió la germinación de manera variable a las semillas de distinta procedencia.

Distintos estudios han mencionado que el peso de las semillas influencía varios atributos de las plantas, desde la dispersión potencial a la persistencia en bancos de semillas y los índices de crecimiento de plántulas (Fenner \& Thompson 2005, Walck et al. 2011). Nosotros encontramos una alta variabilidad en el peso de las semillas de $C$. edentula a lo largo de la costa norte de la Península de Yucatán (Tabla 1), y los resultados indican que las semillas son de menor peso que las reportadas para la costa del Atlántico en Norteamérica (7.9 \pm 2.5 mg; Maun \& Payne 1989). En nuestro estudio, las semillas más pequeñas y con mayor contenido de humedad, que fueron colectadas en la localidad de El Cuyo, fueron las semillas con los mayores porcentajes de germinación. Es posible que las plántulas de $C$. edentula que emergen en las dunas costeras de este sitio, tendrían una mayor probabilidad de establecerse debido a que los niveles de precipitación son más altos que en los de otros sitios estudiados. Una mayor germinación en oscuridad sería crucial para las plántulas ya que podrían comenzar su desarrollo en profundidades mayores dentro de la arena, en donde la humedad es alta (Tobe et al. 2006). No obstante, se ha mencionado que en especies que habitan la primera línea de costa, un mayor tamaño (peso) de las semillas puede ser de significancia adaptativa. Maun \& Payne (1989) mencionan que la dinámica de la duna costera podría ser un importante agente de selección del peso de las semillas en especies de Cakile ya que, al ser transportada a una distancia incierta con alta probabilidad de enterramiento en las dunas de arena, las semillas con tamaños más grandes serían favorecidas.

La germinación de semillas es un elemento clave de la respuesta de las plantas a su ambiente, y la variación en las estrategias de germinación de semillas es comúnmente observada entre especies (Baskin \& Baskin 1998, Barga et al. 2017). En nuestro estudio, independientemente del sitio de procedencia, las semillas de $C$. edentula tuvieron valores de viabilidad similares (30-60\%), no presentaron latencia y germinan rápidamente en oscuridad y con humedad constante. A pesar de ello, el porcentaje de germinación final varió entre sitios de procedencia entre 20 y $100 \%$ en condiciones de oscuridad, y 0-50 \% en presencia de luz (Figuras 1, 2).

En relación con las variables bioclimáticas de los sitios de procedencia de semillas, los sitios de El Cuyo y Holbox 
Tabla 4. Coeficiente de correlación de Pearson (r) de la asociación entre los atributos de las semillas de Cakile edentula y de las variables bioclimáticas de los sitios de procedencia en la Península de Yucatán, México.

\begin{tabular}{lccccccc}
\hline \multirow{2}{*}{ Atributo } & \multicolumn{9}{c}{ Variable bioclimática } \\
\cline { 2 - 8 } & Bio5 & Bio8 & Bio9 & Bio11 & Bio13 & Bio14 & Bio19 \\
\hline Peso (mg) & $-.42 \mathrm{~ns}$ & $.37 \mathrm{~ns}$ & $.41 \mathrm{~ns}$ & $.40 \mathrm{~ns}$ & $.13 \mathrm{~ns}$ & $.16 \mathrm{~ns}$ & $-.34 \mathrm{~ns}$ \\
CH (\%) & $.56 \mathrm{~ns}$ & $-.64 \mathrm{~ns}$ & $-.74 \mathrm{~ns}$ & $-.75 \mathrm{~ns}$ & $.36 \mathrm{~ns}$ & .60 & .76 \\
Germinación (\%) & $.82^{*}$ & $-.47 \mathrm{~ns}$ & $-.77 \mathrm{~ns}$ & $-.81^{*}$ & $.36 \mathrm{~ns}$ & $.33 \mathrm{~ns}$ & $.80^{*}$ \\
Viabilidad & $.00 \mathrm{~ns}$ & $-.62 \mathrm{~ns}$ & $-.49 \mathrm{~ns}$ & $-.44 \mathrm{~ns}$ & $-.54 \mathrm{~ns}$ & $.01 \mathrm{~ns}$ & $.04 \mathrm{~ns}$ \\
\hline
\end{tabular}

$*=P<0.05, \mathrm{~ns}=$ no significativo.

se diferenciaron de los demás por presentar precipitaciones más altas en el trimestre más frio y en el más seco. En estos sitios de procedencia se colectaron las semillas con mayor capacidad germinativa. Sin embargo, solamente se encontró una asociación positiva y significativa entre el porcentaje de germinación de las semillas y la temperatura máxima del mes más cálido y con la precipitación del trimestre más frío (Tabla 4). Los resultados sugieren que existe una mayor capacidad germinativa en semillas de C. edentula en sitios donde la precipitación es mayor en los periodos de menor temperatura dentro de su rango de distribución. Contrario a nuestros resultados, en un estudio similar en la costa de Oaxaca, Viveros-Viveros et al (2017) encontraron una asociación positiva y significativa entre la capacidad germinativa de Enterolobium cyclocarpum con la proporción de precipitación que cae durante el verano y entre la germinación media diaria y la precipitación total que ocurre en la estación de crecimiento. La capacidad germinativa de esa especie varió de 28.2 a 90 $\%$, dependiendo del sitio de procedencia.

La variabilidad fenotípica de los atributos de las semillas entre sitios de procedencia puede incrementar la resiliencia de las especies y reducir el riesgo de extinción bajo cambio climático. Los resultados de nuestro estudio sugieren que el peso de las semillas y la capacidad de germinación podrían ser los atributos de las semillas más sensibles al cambio ambiental entre sus poblaciones. No obstante, para resolver esta interrogante sería necesario estimar si los distintos escenarios de cambio climático podrían limitar la regeneración en las poblaciones de $C$. edentula. Estimar la capacidad germinativa dentro de un gradiente de temperatura experimental, así como evaluar la sobrevivencia de semillas y plántulas de distinta procedencia sería crucial para identificar los atributos y las poblaciones con mayor riesgo.

\section{Agradecimientos}

Esta investigación fue apoyada por la beca postdoctoral DGAPA-UNAM de Ricardo Álvarez. Agradecemos el soporte brindado por el Dr. Alfonso Larqué y el Banco de Germoplasma del Centro de Investigaciones Científicas de Yucatán, A. C. para la realización de las pruebas de laboratorio, así como al M. en C. Johnny Valdez por su apoyo en el trabajo de campo. Se agradece a los revisores ya que con sus comentarios y sugerencias permitieron mejorar el manuscrito.

\section{Literatura citada}

Barga S, Dilts TE, Leger EA. 2017. Climate variability affects the germination strategies exhibited by arid land plants. Oecologia 185: 437-452.

DOI: http://doi.org/10.1007/s00442-017-3958-5

Baskin CC, Baskin JM. 1998. Seeds. Ecology, biogeography and evolution of dormancy and germination. San Diego: Academic Press. ISBN: 978-0-12-080260-9

Carta A, Probert R, Puglia G, Peruzzi L, Bedini G. 2016. Local climate explains degree of seed dormancy in Hypericum elodes L. (Hypericaceae). Plant Biology 18: 76-82. DOI: http://doi.org/10.1111/plb.12310

Cochrane A, Daws MI, Hay FR. 2011. Seed-based approach for identifying flora at risk from climate warming. Austral Ecology 36: 923-935.

DOI: http://doi.org/10.1111/j.1442-9993.2010.02211.x

Cochrane A, Yates CJ, Hoyle GL, Nicotra AB. 2015. Will among-population variation in seed traits improve the chance of species persistence under climate change? Global Ecology and Biogeography 24: 12-24.

DOI: http://doi.org/10.1111/geb.12234.

Donohue K. 1998. Maternal determinants of seed dispersal in Cakile edentula: fruit, plant, and site traits. Ecology 79: 27712788. DOI: http://doi.org/10.1890/0012-9658(1998)079[2771:MDOSDI]2.0.CO;2.

Fenner M, Thompson K. 2005. The ecology of seeds, 2 ed. Cambridge University Press: Cambridge. ISBN: 9780521653688

Fernández-Pascual E, Jiménez-Alfaro B, Hájek M, Díaz TE, Pritchard HW. 2015. Soil thermal buffer and regeneration niche may favour calcareous fen resilience to climate change. Folia Geobotanica 4: 293-301.

DOI: http://doi.org/10.1007/s12224-015-9223-y.

García E. 2004. Modificaciones al sistema de clasificación climática de Köppen. México DF: Serie libros. Instituto de Geografía-UNAM. ISBN: 970-32-1010-4

Guo H, Mazer SJ, Du G. 2010. Geographic variation in seed mass within and among nine species of Pedicularis (Orobanchaceae): effects of elevation, plant size and seed number per fruit. Journal of Ecology 98: 1232-1242. DOI: http://doi.org/10.1111/j.1365-2745.2010.01688.x.

Hong TD, Ellis RH. 1996. A protocol to determine seed storage behaviour (No. 1). Bioversity International. ISBN 92-9043279-9 
ISTA [International Seed Testing Association]. 2015. International rules for seed testing, 2015: 1-276.

DOI: http://doi.org/10.15258/istarules.2015.f

Jiménez-Alfaro B, Silveira FAO, Fidelis A, Poschlod P. 2016. Seed germination traits can contribute better to plant community ecology. Journal of Vegetation Science 27: 637-645. DOI: http://doi.org/10.1111/jvs.12375

Jiménez-Orocio O, Espejel I, Martínez ML. 2015. La investigación científica sobre dunas costeras de México: origen, evolución y retos. Revista Mexicana de Biodiversidad 86:486507. DOI: http://doi.org/10.1016/j.rmb.2015.04.022

Maun MA, Payne AM. 1989. Fruit and seed polymorphism and its relation to seedling growth in the genus Cakile. Canadian Journal of Botany 67: 2743-2750.

DOI: http://doi.org/10.1139/b89-353.

Maun M, Boyd RS, Olson L. 1990. The Biological Flora of Coastal Dunes and Wetlands. 1. "Cakile Edentula" (Bigel.) Hook. Journal of Coastal Research 6: 137-156.

Rosbakh S, Poschlod P. 2015. Initial temperature of seed germination as related to species occurrence along temperature gradient. Functional Ecology 29: 5-14. DOI: http://doi.org/10.1111/1365-2435.12304.

Thompson K, Ceriani RM. 2003. No relationship between range size and germination niche width in the UK herbaceous flora. Functional Ecology 17: 335-339.

DOI: http://doi.org/10.1046/j.1365-2435.2003.00734.x.

Tobe K, Zhang L, Omasa K. 2006. Seed germination and seedling emergence of three Artemisia species (Asteraceae) inhabiting desert sand dunes in China. Seed Science Research 16: 61-69. DOI: http://doi.org/10.1079/SSR2005230

Torres W, Méndez M, Dorantes A, Durán R. 2010. Estructura, composición y diversidad del matorral de duna costera en el litoral yucateco. Boletín de la Sociedad Botánica de México

Editor de sección: Enrique Jurado.

Contribuciones de los autores: Ricardo Álvarez concibió la idea, realizó el diseño del experimento, analizó los datos y escribió el artículo. Gabriela Mendoza analizó los datos y desarrolló la metodología. Candelaria Pérez concibió la idea y desarrolló la metodología. Xavier Chiappa coordinó la investigación y revisó la versión final del manuscrito.
86: $37-51$.

Torres-Martínez. L, Weldy P, Morris L, Emery NC. 2017. Spatiotemporal heterogeneity in precipitation patterns explain population-level germination strategies in an edaphic specialist. Annals of Botany 119: 253-265.

DOI: http://doi.org/10.1093/aob/mcw161.

Venable DL, Brown JS. 1988. The selective interactions of dispersal, dormancy, and seed size as adaptations for reducing risk in variable environments. The American Naturalist 131: 360-384. DOI: http://doi.org/10.1086/284795.

Viveros-Viveros H, Quino-Pascual K, Velasco-García MV, Sánchez-Viveros G, Velasco-Bautista E. 2017. Variación geográfica de la germinación en Enterolobium cyclocarpum en la costa de Oaxaca, México. Bosque 38: 317-326. DOI: http://doi.org/10.4067/S0717-92002017000200009

Walck JL, Hidayati SN, Dixon KW, Thompson K, Poschlod P. 2011. Climate change and plant regeneration from seed. Global Change Biology 17: 2145-2161.

DOI: http://doi.org/10.1111/j.1365-2486.2010.02368.x.

Westoby M, Falster DS, Moles AT, Vesk PA, Wright IA. 2002. Plant ecological strategies: some leading dimensions of variation between species. Annual Review of Ecology and Systematics 33: 125-159. DOI:

http://doi.org/10.1146/annurev.ecolsys.33.010802.150452.

Wu H, Meng H, Wang S, Wei X, Jiang M. 2018. Geographic patterns and environmental drivers of seed traits of a relict tree species. Forest Ecology and Management 422: 59-68. DOI: http://doi.org/10.1016/j.foreco.2018.04.003.

Zani D, Müller JV. 2017. Climatic control of seed longevity of Silene during the post-zigotic phase: do seeds from warm, dry climates possess higher maturity and desiccation tolerance than seeds from cold, wet climates? Ecological Research 32: 983-994. DOI: http://doi.org/10.1007/s11284-017-1508-6. 